\title{
Surgical Prehabilitation: Systematic Review
}

\author{
Andrés Armendariz Rodríguez ${ }^{1 *}$, Lourdes Rocío Álvarez López ${ }^{2}$, Alberto Robles Méndez \\ Hernández ${ }^{2}$, Laura Paulina Baena Hernández ${ }^{1}$, Erika Bautista Rodríguez ${ }^{1}$, Rodrigo Dominic \\ Cerqueda Audirac ${ }^{1}$, Ariadna Miranda Alvarado Benítez ${ }^{1}$ \\ ${ }^{1}$ Medical intern, Universidad Nacional Autónoma de México, Mexico City, Mexico \\ ${ }^{2}$ Department of General Surgery, Hospital Angeles Metropolitano, Mexico City, Mexico
}

*Corresponding Author: Alberto Robles Méndez Hernández, General Surgery Service, Hospital Ángeles Metropolitano, Tlacotalpan \#59, Mexico City, Mexico.

\begin{abstract}
Prehabilitation is focused on improving the patient's functioning capabilities before undergoing surgical treatment.There exist many recommendations about pre-habilitation, such as weight loss, nutritional support, and physical exercise following the FITT model. These recommendations are decisive on the functional capacity, and not modifying them can result in risk factors for an inadequate response to the surgical treatment and future complications.
\end{abstract}

Objective and Methods: The search was performed on 2 search engines: PubMed and the Cochrane library. The criteria the articles had to fulfill in order to be included in the review were: language English and no older than 5 years, after reviewing analysis methodology and quality of articles, 18 articles from PubMed, and 15 from the Cochrane Library were included.

Conclusions: Given that there exists studies with diverse results on the benefit of pre-habilitation, it is necessary to perform studies with higher samples in order to be able to obtain conclusive outcomes and turn pre-habilitation into a strategy that can be reproducible at the majority of the hospital centers.

Keywords: Abdominal Surgery, Prehabilitation, Thoracic Surgery

\section{INTRODUCTION}

Pre-habilitation is focused on improving the patient's functioning capabilities before undergoing surgical treatment. A quicker recovery from a major surgery does not only depend on the surgical process itself, but also the hygienic-dietetic and psychological measurements applied on the patients before their surgery.

Despite the great advancements that have been achieved on surgical techniques, anesthesia, analgesia, and perioperative attentions, morbimortality and the post-major surgery complications rate stayed above $30 \%$, with a physical reserve reduction of a 40\%.[1]

Given that elective surgery is a "scheduled stress", it is important to improve the organism's responses to that stress through prehabilitation, defined as a multi-disciplinary intervention, which objective is to take advantage of pre-operational period to improve the individual's functional capacity by implementing hygienic-dietetic and psychological measurements, and in this way, to favor an early post-surgical recuperation, as well as prevent or attenuate the functional impairment related to the surgery and its consequences. $[2,3]$

\section{2. ОвJECTIVE}

There have been implemented programs aimed to apply a pre-habilitation process on patients to help them reach an optimal post-surgical recovery throughout exercise, nutritional and psychological attention. However, very little has been systematically developed to improve the patient's functional capacity previous to surgical intervention.

Due to the growing amount of evidence on the effectiveness of surgical pre-habilitation, this review will identify the most relevant preoperative interventions, proposing in a more specific way, a clinical pathway that is 
structured to improve the doctor's preventive and therapeutic capacity.

\section{Methodology}

The search was performed on 2 search engines: PubMed and the Cochrane library.

The criteria the articles had to fulfill in order to be included in the review were:

English Language

No older than 5 years

The search on PubMed was carried out using the term "Surgical Prehabilitation", with "5 years" as a filter. 26 ' 438 results were found. Figure 1

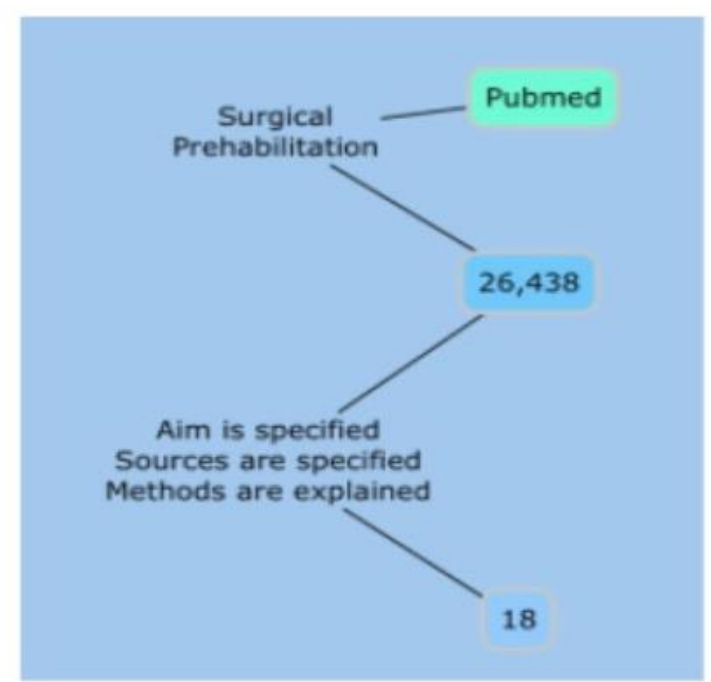

Figure 1; Results in PubMed

\section{DISCUSSION}

It has been proven that surgical pre-habilitation has diminished the morbimortality rate on different types of major surgeries.

When modifying metabolic, sarcopenia, and psychological stress risk factors before surgical intervention, post-surgical complications have been diminished.

Many ways of attenuating these modifiable risk factors have been tried. Some of them, unsuccessfully.[4]

\section{Some of the interventions that have been carried out are}

\section{Nutrition:}

The nutritional state before the surgical event is directly tied to its result. Preoperative nutritional optimization aims to address the following goals:

- Optimize nutrient stores
On Cochrane Library, the terms "Prehabilitation And Surgery" were used, "And" serving as a Boolean operator. The search gave 292 results. Figure 2

The quality of the articles was evaluated, considering:

The article's objective being specified

Sources being identified

The analysis' methodology being explained

The references' antiqueness

Once the articles' quality was evaluated, 18 articles from PubMed, and 15 from the Cochrane Library were included.

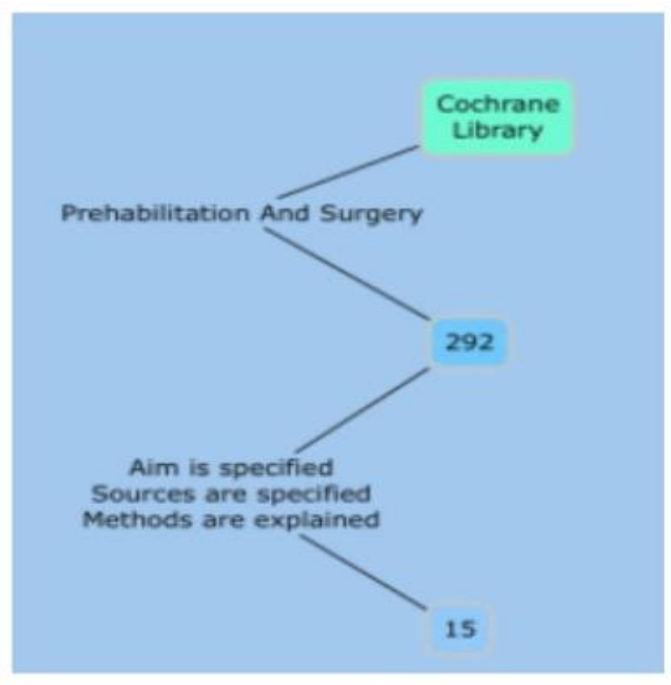

Figure 2; Results in Cochrane Library

- Optimize metabolic reserve

- Provide a buffer to trauma-induced catabolism

- Enhance immune response

- Provide a nutritional strategy for the entire perioperative period

- Target those at the highest risk

The BMI and TBW are the most common indicators of a bad nutritional state, but actually, these values could be altered by different factors. Thus, the Perioperative Nutrition Screening (PONS) was developed.

PONS

- Step $1 \mathrm{BMI}<18.5(<20$ if the patient is older than 65 years old)

- Step 2 Unintentional weight loss $>10 \%$ in the past six months 
- Step 3 During the last week, you've eaten at least $50 \%$ less than what you usually eat

If the patient feels identified with any of these three statements, or if their Albumin levels are $<3.0$, they must consult a nutrition specialist.

The intake of carbohydrate-rich beverages 24 before the surgical intervention is recommended.[5]

\section{Exercise:}

The FITT (frequency, intensity, time, type) model is recommended for the planning of an exercise routine that's customized for every patient, depending on their age and physical condition.

The American College of Sports Medicine claims that exercises, such as walking 15 minutes a day for increasing a person's heart rate, have the same benefit as the most exhaustive training methods.[6]

\section{Psychologic factors on the surgical pre- habilitation:}

Usually, surgical pre-habilitation has been centered on physical comorbidities, such as diabetes, anemia, diet optimization, and physical activity/exercise. Just recently, there has been a growing recognition of the importance of psychological morbidity. Evidence suggests that psychological factors influence the surgical, physical, and psychological results in both, the short and long terms. All of this has given place to the emergence of psychological prehabilitation and the trimodal focus, which incorporates psychological intervention, exercise, and nutritional optimization. When working, anxiety and depression are the predominant topics to be addressed, as well as the improvement of coping abilities.[7]

\section{Psychologic factors and their relation to post- surgical results:}

Some psychological characteristics have a protective effect, while other ones have been associated with a negative effect.

Factors associated with favorable results: Selfefficacy, low pain expectation, external locus of control, optimism, religiousness, anger control.

Factors associated with unfavorable results: Trait anxiety, state anxiety, depression, intramarital hostility, state anger, psychological distress.[7]

Rosenberger et al. evaluated the effect of the mood, attitudinal factors, personality and coping mechanisms on the complications, the pain, the use of analgesics, functional recovery, the duration of the hospital stay, and the qualification of the physical recuperation.[8]

Speaking about mood, anxiety foretold the patient's short-term surgical results, the duration of their hospital stay, and their recuperation qualifications. Depression foretold the long-term pain. Attitudinal factors, such as the patient's self-efficacy, positivity, and perceived control were associated with a quicker functional recuperation. Personality measures, such as neuroticism and extroversion, were not strong foretellers of short-term physical results.

\section{Psychological factors and postoperative pain:}

The relation between psychological factors and post-surgical pain has been studied by various authors. $[9,10]$

Ip et al. identified preoperative factors that foretold the postoperative pain. Anxiety was one of the four factors that were found to be reliably associated with postoperative pain. The other ones are age, type of surgery, and preoperative pain.

The systematic revisions provide a high level of evidence of the predictive value of pre-surgical depression, psychological vulnerability, and chronic stress on the risk of chronic pain after the surgery.[11]

\section{Special Recommendations}

\section{Thoracic Surgery:}

Performing aerobic and deep breathing exercises is recommended.

It's also recommended to lose two kilograms before the scheduled surgery.

During a clinical trial, the patients who performed deep breathing exercises and lost two kilograms two weeks before surgical intervention had a shorter hospital stay (three days) than the patients who did not undergo any intervention.[12]

\section{Bariatric Surgery:}

It's recommended to educate the patient. 
During a clinical trial, it was observed that patients who received education on nutrition and physical training before the surgical intervention, had a better result on weight loss after 4 months. This tendency was maintained for the 6- and 8-month evaluations.[13]

\section{Abdominal Surgery:}

It is recommended to perform the nutritional evaluation six weeks before, as well as doing aerobic and breathing exercises to lower the post-surgical morbidity.

During a clinical trial, patients who received nutritional evaluation six weeks before their surgical event had a lower amount of complications in comparison to those patients who did not undergo any intervention.[14]

\section{Inspiratory Muscle Training:}

Inspiratory Muscle Training (IMT) consists of training the respiratory muscles' strength and resistance, generally with an intensity gradation, starting with a 20 to $30 \%$ of the maximum inspiratory pressure, and progressing until the $60 \%$.[15] Generally, the level of evidence for the preoperatory IMT for fragile patients is very low, due to the lack of studies.[16]

\section{CONClusions}

With the review that has been performed, it concludes that the success of a quicker and a complete recovery from a major surgery does not only depend on the surgical process itself, but also the hygienic-dietetic and psychological measurements applied on the patients before their surgery.

Likewise, it concludes that as pre-habilitation is a set of multi-disciplinary measurements, it is important to consider every recommendation coming from the expert area, needing more studies for the creation of a systematic consensus about the measurements to be applied on the different kinds of surgeries and on the different age groups, as well as specifying the recommended optimal time, and the goals that must be achieved before the surgery.

Given that there exists studies with diverse results on the benefit of pre-habilitation, it is necessary to perform studies with higher samples in order to be able to obtain conclusive outcomes and turn pre-habilitation into a strategy that can be reproducible at the majority of the hospital centers.

\section{FUNDING \& CONFLICTS OF INTEREST}

None

\section{ETHICAL APPROVAL}

There was no ethics approval required for this systematic review.

\section{REFERENCES}

[1] Lucas DJ, Pawlik TM. Quality improvement in gastrointestinal surgical oncology with American College of Surgeons National Surgical Quality Improvement Program. Surgery. 2014 Apr;155(4):593-601.

[2] Minnella EM, Carli F. Prehabilitation and functional recovery for colorectal cancer patients. Eur J Surg Oncol. 2018 Jul;44(7):919_ 26.

[3] Carli F, Scheede-Bergdahl C. Prehabilitation to Enhance Perioperative Care. Anesthesiol Clin. 2015 Mar;33(1):17-33.

[4] Shem Tov L, Matot I. Frailty and anesthesia: Curr Opin Anaesthesiol. 2017 Jun;30(3):40917.

[5] Blitz JD, Mabry C. Designing and Running a Preoperative Clinic. Anesthesiol Clin. 2018 Dec;36(4):479-91.

[6] Bingener J, Cutshall SM, Skaran PE, et al Patient-centered surgical prehabilitation. Am J Surg. 2019 Sep;218(3):666.

[7] Levett DZH, Grimmett C. Psychological factors, prehabilitation and surgical outcomes: evidence and future directions. Anaesthesia. 2019 Jan;74:36-42.

[8] Rosenberger PH, Jokl P, Ickovics J. Psychosocial Factors and Surgical Outcomes: An Evidence-Based Literature Review: J Am Acad Orthop Surg. 2006 Jul;14(7):397-405.

[9] Dadgostar A, Bigder M, Punjani N, et al. Does preoperative depression predict post-operative surgical pain: A systematic review. Int J Surg. 2017 May;41:162-73.

[10] Weinrib AZ, Azam MA, Birnie KA, et al. The psychology of chronic post-surgical pain: new frontiers in risk factor identification, prevention and management. $\mathrm{Br} \quad \mathrm{J}$ Pain. 2017 Nov;11(4):169-77.

[11] Hinrichs-Rocker A, Schulz K, Järvinen I, et al. Psychosocial predictors and correlates for chronic post-surgical pain (CPSP) - A systematic review. Eur J Pain. 2009 Aug;13(7):719-30.

[12] Barberan-Garcia A. Cost-effectiveness of a Multimodal Prehabilitation Program in Highrisk Patients Undergoing to Lung Resection: A Randomized Clinical Trial [Internet]. clinicaltrials.gov; 2019 Dec [cited 2020 Aug 13]. Report No.: NCT04052100. Available 
from:

https://clinicaltrials.gov/ct2/show/NCT0405210 0

[13] Prehabilitation in Bariatric Surgery - Full Text View - ClinicalTrials.gov [Internet]. [cited 2020 Aug 16]. Available from: https://clinicaltrials.gov/ct2/show/NCT0404636 7

[14] VA Office of Research and Development. Pilot Testing Prehabilitation Services Aimed at Improving Outcomes of Frail Veterans Following Major Abdominal Surgery [Internet]. clinicaltrials.gov; 2020 Mar [cited 2020 Aug
13]. Report No.: NCT03040336. Available from:

https://clinicaltrials.gov/ct2/show/NCT0304033 6

[15] Tew GA, Ayyash R, Durrand J, et al. Clinical guideline and recommendations on preoperative exercise training in patients awaiting major non-cardiac surgery. Anaesthesia. 2018 Jun;73(6):750-68.

[16] Milder DA, Pillinger NL, Kam PCA. The role of prehabilitation in frail surgical patients: A systematic review. Acta Anaesthesiol Scand. 2018 Nov;62(10):1356-66.

Citation: Andrés Armendariz Rodríguez, Lourdes Rocío Álvarez López, Alberto Robles Méndez Hernández, Laura Paulina Baena Hernández, Erika Bautista Rodríguez, Rodrigo Dominic Cerqueda Audirac, Ariadna Miranda Alvarado Benítez. Surgical Prehabilitation: Systematic Review. ARC Journal of Surgery. 2020; 6(2):9-13. DOI: https://doi.org/ 10.20431/2455-572X.0602002.

Copyright: (C) 2020 Authors. This is an open-access article distributed under the terms of the Creative

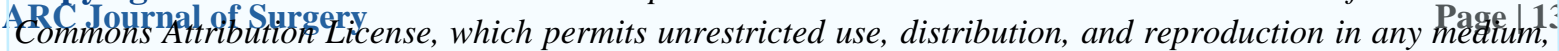
provided the original author and source are credited. 